\title{
ON THE RELATION BETWEEN MASS AND CHARGE: A Pure Geometric Approach.
}

\begin{abstract}
M.I.Wanas ${ }^{1}$
Abstract

A new solution of the field equations of the generalized field theory, constructed by Mikhail and Wanas in 1977, has been obtained. The geometric structure used, in the present application, is an absolute parallelism (AP)-space with spherical symmetry (type FIGI). The solution obtained represents a generalized field outside a charged massive central body. Two schemes have been used to get the physical meaning of the solution: The first is related to the metric of the Riemannian space associated with the AP-structure. The second is connected to a covariant scheme known as Type Analysis. It is shown that the dependence on both schemes for interpreting the results obtained, is more better than the dependence on the metric of the Riemannian space associated with the AP-structure.

In General, if we consider the solution obtained as representing a geometric model for an elementary charged particle, then the results of the present work can be summarized in the following points. (i) It is shown that the mass of the particle is made of two contributions: The first is the gravitational contribution, and the second is the contribution due to the existence of charge. (ii) The model allows for the existence of a charged particle whose mass is completely electromagnetic in origin. (iii) The model prevents the existence of a charged massless particle. (iv) The electromagnetic contribution, to the mass, is independent of the sign of the electric charge. (v) It is shown that the mass of the electron (or a positron) is purely made of its charge.
\end{abstract}

\section{Introduction}

In field theories, solutions with spherical symmetry have received much attention, since they simplify several physical and astrophysical problems. In the theory of general relativity, these solutions may be used as models for static (or slowly rotating) celestial objects with no net electric charge. While in Einstein-Maxwell's theory they represent models for static objects with non-vanishing net electric charge.

The formation of a stable charged particle, in the framework of classical electrodynamics, is impossible. This is due to the fact that the field of an electric charge with a certain sign is repulsive. Many authors believe that the best classical description of elementary particles is that of singularities in a combined electromagnetic-gravitational field (cf. [1]). Others have the hope to explain the masses of charged particles by reducing them to the energy of their electromagnetic fields [2]. This may lead to some developments in the theory of elementary particles. A comprehensive theory of gravity and electromagnetism may be very useful in discussing this problem, since the existence of a gravitational field (attractive) together with an electromagnetic field (repulsive) may lead to a stable charged particle.

\footnotetext{
${ }^{1}$ Astronomy Department, Faculty of Science, Cairo University, Giza, Egypt.

E-mail:wanas@frcu.eun.eg
} 
The main problem with Einstein-Maxwell's theory is that the gravitational field and the electromagnetic field are treated asymmetrically. The gravitational field has been geometrized while the electromagnetic field is introduced as a vector field added to the symmetric tensor field $g_{\mu \nu}$ of Riemannian geometry. There is no definite role for the added field in the original geometry.

In the generalized field theory (GFT) [3], constructed by Mikhail and Wanas in 1977,using Absolute Parallelism (AP)-geometry, the two fields have been treated, in a comprehensive way, as one entity. All geometric elements, used to describe gravitational and electromagnetic quantities, are derived directly from the geometric structure used. The theory has been applied to spherically symmetric cases [4],[5], [6]. The solutions obtained, so far, were found to be in agreement with previously known results, under some limiting (particular) conditions.

The aim of the present work is to explore a further solution, with spherical symmetry. This may throw some light on the previously stated problems. In section 2 the bases of the geometric structure used for application is presented. A brief review of the GFT, the version of AP-geometry used in its construction and a summary of its applications are given in section 3. A new solution of the field equation, of the GFT, is obtained in section 4. Some of the electromagnetic and gravitational quantities are evaluated in section 5 . The results are discussed in section 6 and the paper is concluded in section 7 .

\section{Summary of a Version of AP-geometry}

In the present section, a brief review of the conventional AP-geometry, first used by Einstein [7] and developed by Robertson [8] and Mikhail [9], is given. The geometry used to construct the GFT is a version AP-geometry. An AP-space is an affinely connected space, each point of which is labeled by a set of $n$-independent variables $x^{\nu}(\nu=1,2,3, \ldots . n)$. At each point we introduce n-linearly independent contravariant vectors $\lambda^{\nu}(i=1,2,3, \ldots . n)$. We denote by $\underset{i}{\lambda_{\mu}}$ the normalized co-factor of $\underset{i}{\lambda^{\mu}}$ in the determinant of ${ }_{i}^{i}$ so that,

$$
\begin{gathered}
\lambda_{i}^{\mu}{ }_{i}{ }_{i}=\delta_{\nu}^{\mu}, \\
\lambda_{i}^{\mu}{ }_{j}{ }_{j}=\delta_{i j},
\end{gathered}
$$

where summation convention is carried out over repeated indices, regardless of their positions. The affine connection $\Gamma_{\mu \nu}^{\alpha}$ characterizing the space will be defined as a consequence of absolute parallelism condition,

$$
{\underset{i}{+}+\nu}_{+\mu}^{\mu}=0
$$

where the $(+)$ sign and the stroke used to characterize this type of tensor differentiation using the affine connection $\Gamma_{\mu \nu}^{\alpha}$. For a space of dimension 4 , there are 64 equations (3) with the unique solution,

$$
\Gamma_{\mu \nu}^{\alpha}=\underset{i}{\lambda}{ }_{i}^{\alpha} \lambda_{\mu, \nu}
$$


where the comma is used for ordinary partial differentiation. The curvature tensor corresponding to this connection,

$$
M_{. \mu \nu \sigma}^{\alpha} \stackrel{\text { def }}{=} \Gamma_{. \mu \sigma, \nu}^{\alpha}-\Gamma_{\cdot \mu \nu, \sigma}^{\alpha}+\Gamma_{. \mu \sigma}^{\epsilon} \Gamma_{. \epsilon \nu}^{\alpha}-\Gamma_{. \mu \nu}^{\epsilon} \Gamma_{. \epsilon \sigma}^{\alpha}
$$

vanishes identically, while that corresponding to its dual $\tilde{\Gamma}_{\mu \nu}^{\alpha}\left(=\Gamma_{\nu \mu}^{\alpha}\right)$ does not vanish (a detailed geometric study of the curvature tensors in the AP-space has been recently given [10]). Tensor differentiation using $\tilde{\Gamma}_{\mu \nu}^{\alpha}$ is distinguished by a (-)sign. The torsion tensor, of the connection (4), is usually defined by,

$$
\Lambda_{. \mu \nu}^{\alpha} \stackrel{\text { def }}{=} \Gamma_{. \mu \nu}^{\alpha}-\Gamma_{. \nu \mu}^{\alpha}
$$

Another third order tensor (contortion) is defined by,

$$
\gamma_{. \mu \nu}^{\alpha} \stackrel{\text { def }}{=} \underset{i}{\lambda_{i}^{\alpha}} \underset{i}{\lambda} \mu ; \nu
$$

where the semicolon is used to characterize covariant differentiation using Christoffel symbol. The two tensors are related by the formula,

$$
\gamma_{. \mu \nu}^{\alpha}=\frac{1}{2}\left(\Lambda_{. \mu \nu}^{\alpha}-\Lambda_{\nu . \mu}^{\alpha}-\Lambda_{\mu . \nu}^{\alpha}\right)
$$

A basic vector could be obtained by contraction of one of the above third order tensors, i.e.

$$
C_{\mu} \stackrel{\text { def }}{=} \Lambda_{. \mu \alpha}^{\alpha}=\gamma_{. \mu \alpha}^{\alpha} .
$$

Using the building blocks of the AP-space one can define the following second order symmetric tensor,

$$
g_{\mu \nu} \stackrel{\text { def }}{=} \underset{i}{\lambda_{\mu}}{ }_{i} \lambda_{\nu}
$$

This tensor is used as a metric tensor of the Riemannian space, associated with the AP-space, when needed.

The following table contains second order tensors, defined in the AP-space, that are used in most physical applications. It has been originally constructed by Mikhail [7]. 
Table 1: Second Order World Tensors [7]

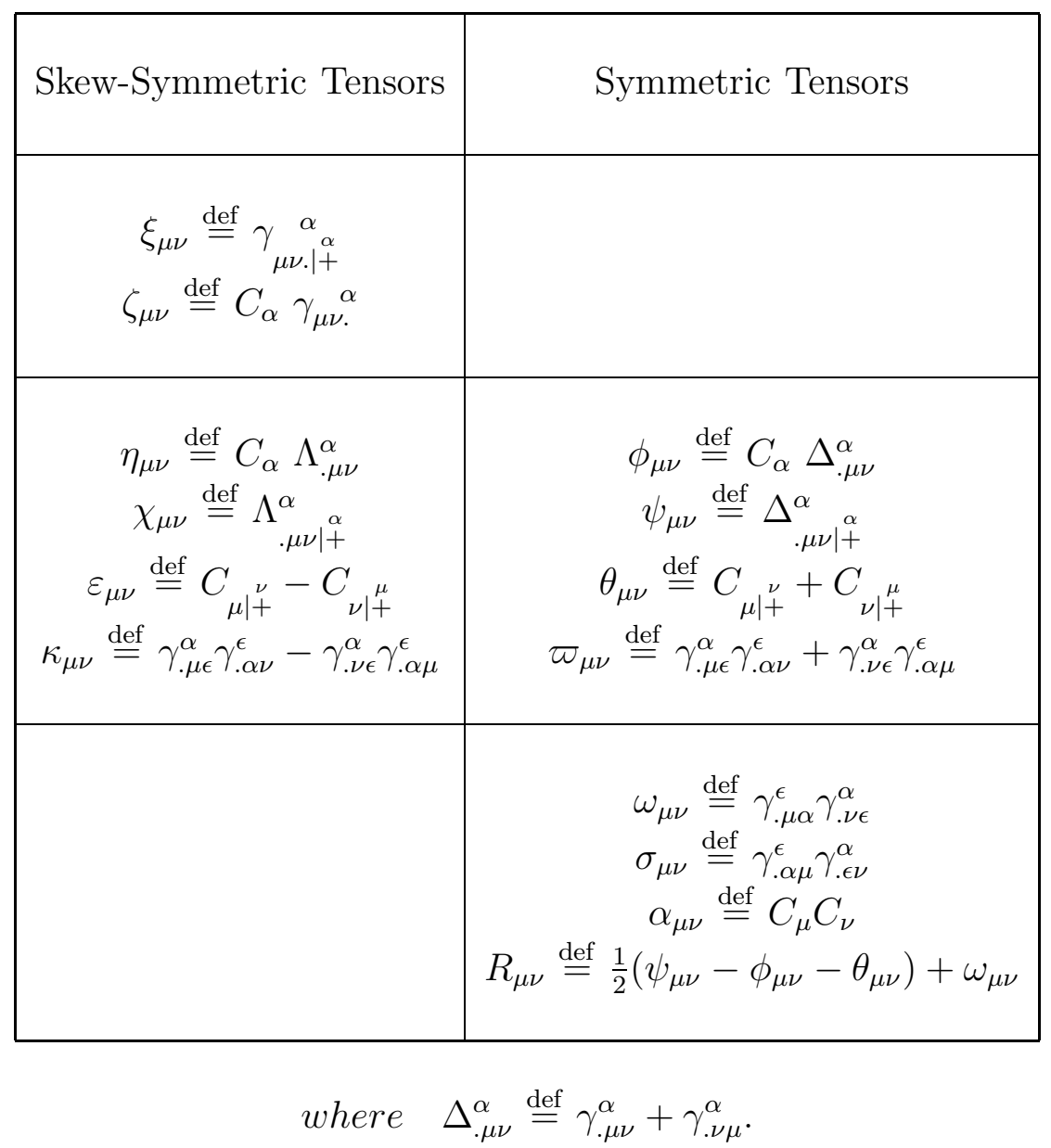

\section{A Brief Review of GFT: Bases and Results}

The Generalized Field Theory is a pure geometric attempt to unify gravity and electromagnetism. It has been constructed in 1977 by Mikhail and Wanas [3]. In general, the theory is a covariant one. Its anti-symmetric section, representing electromagnetism in the weak field regime, is gauge invariant. The underlaying geometry, for this theory, is a version of the AP geometry. This version is slightly different from that usually used in the literature, since it is based on the dual connection and is of the Riemann-Cartan type. The main advantages of this version are clarified in the following lines. Consider the tensor derivative, of an arbitrary covariant vector $A_{\mu}$, using the dual connection,

$$
A_{-\mid \nu} \stackrel{\text { def }}{=} A_{\mu, \nu}-A_{\alpha} \tilde{\Gamma}_{. \mu \nu}^{\alpha}
$$

The commutation formula of this type of differentiation, for an arbitrary covariant vector, can be expressed by the following relation,

$$
A_{-\mid \nu \sigma}-A_{-\mid \sigma \nu}=A_{\alpha} \tilde{M}_{. \mu \nu \sigma}^{\alpha}-A_{-\mid \alpha} \tilde{\Lambda}_{. \nu \sigma}^{\alpha},
$$


where,

$$
\tilde{M}_{. \mu \nu \sigma}^{\alpha} \stackrel{\text { def }}{=} \tilde{\Gamma}_{\cdot \mu \sigma, \nu}^{\alpha}-\tilde{\Gamma}_{\cdot \mu \nu, \sigma}^{\alpha}+\tilde{\Gamma}_{\cdot \mu \sigma}^{\epsilon} \tilde{\Gamma}_{. \epsilon \nu}^{\alpha}-\tilde{\Gamma}_{\cdot \mu \nu}^{\epsilon} \tilde{\Gamma}_{. \epsilon \sigma}^{\alpha}
$$

is the conventional curvature tensor and $\tilde{\Lambda}_{. \nu \sigma}^{\alpha}$ is the torsion tensor corresponding to the dual connection. It is worth of mention that both tensors are, simultaneously, non vanishing in this version. This is another difference between the present version and the AP-version used in the literature. Because of this difference we attribute, to this version of geometry, a Riemann-Cartan character. Now if we replace the covariant vector in equation (12) by the covariant tetrad vectors, then this equation can be written as:

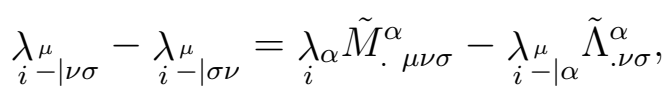

which can be written in the alternative form:

$$
{\underset{i}{\lambda-\mid \nu \sigma}}_{\lambda^{\mu}}-\lambda_{i-\mid \sigma \nu}^{\lambda \mu}=\lambda_{i} \tilde{L}_{. \mu \nu \sigma}^{\alpha},
$$

where $\tilde{L}_{. \mu \nu \sigma}^{\alpha}$ is a fourth order tensor defined by:

$$
\tilde{L}_{. \mu \nu \sigma}^{\alpha} \stackrel{\text { def }}{=} \underset{i}{\lambda_{i}^{\alpha}}\left(\lambda_{i \mu \mid \nu \sigma}-\lambda_{i-\mid \sigma \nu}{ }_{\mu}\right)
$$

We call this tensor the non-conventional curvature tensor. It is a fourth order tensor defined in the present version of the AP-geometry. It is a unique geometric object which measures the non-commutativity of covariant differentiation, using the dual connection. This tensor, implicitly, contains both conventional curvature and torsion tensors. It represents the second main difference between the present version of AP-geometry and the conventional AP-version .

The non-conventional curvature (16) has been used to construct the GFT [3]. The scalar Lagrangian function of the theory is obtained by double contractions of this tensor. Using a certain variational technique, the authors of the theory obtained the differential identity,

$$
E_{. \nu \mid-}^{\mu}=0
$$

Considering this identity as representing a general conservation law, the field equations of the GFT have been written as,

$$
E_{\mu \nu}=0
$$

where,

$$
E_{\mu \nu} \stackrel{\text { def }}{=} g_{\mu \nu} \mathrm{E}-2 L_{\mu \nu}-2 g_{\mu \nu} C_{-\mid \gamma}^{\gamma}-2 C_{\mu} C_{\nu}-2 g_{\mu \alpha} C^{\epsilon} \Lambda_{. \epsilon \nu}^{\alpha}+2 C_{+\mid \mu}-2 g^{\gamma \alpha} \Lambda_{\left.\mu \nu \alpha\right|_{+} ^{\gamma}},
$$

and

$$
\mathrm{E}_{\mu \nu} \stackrel{\text { def }}{=} \Lambda^{\delta}{ }_{\epsilon \mu} \Lambda^{\epsilon} . \delta \nu-C_{\mu} C_{\nu}
$$

This shows that $E_{\mu \nu}$ is a non-symmetric second order tensor defined in the AP-space.

In the following lines, the results of applications of the GFT are summarized.

(i) In the limits of weak static fields and slowly moving test particles [11], the theory gives rise to both Newton's gravity and Maxwell's electromagnetism. Also, calculations in this 
frame shows that Lorentz condition, a condition usually used to fix the gauge in the solutions of Maxwell's equations, is not imposed from outside the GFT, but it arises very naturally as a result of the presence of gravity. The natural appearance of this condition represents a type of interaction between gravity and electromagnetism.

(ii) Using an AP-structure with spherical symmetry, of the type F0GI representing the absence of electromagnetism, the metric of the Riemannian space associated with the solution, of the GFT field equations, is found to be identical to the Schwarzschild exterior metric [4].

(iii) In an application [5], using an AP-structure of the type FIGI, the metric of the Riemannian space associated with the solution obtained is found to be similar to ReissnerNordstrom solution with an extra term. This term tends to zero at large enough distances from the source of the field. In that application, if the constant of integration assigned to the electric charge of the source vanishes, then the metric will reduce to the Schwarzschild metric and the type of the space will be F0GI.

(iv) In a cosmological application of the GFT [12], using an AP-structure of the type F0GIII (representing the absence of electromagnetism and the presence of a strong gravitational field), a unique solution of the field equations has been obtained. This solution represents a pure geometric world model. It is a non-empty expanding model with a density parameter $=0.75$. The model fixes the value of the curvature constant $k=-1$, and is free from particle horizons.

(v) Another application of a solution of the type FIGI, having spherical symmetry, is carried out [6]. This solution shows a new interaction between gravity and electromagnetism. It shows that, a rotating neutral massive object can produce a global magnetic field. The comparison between the theoretical results obtained and astrophysical observations of the magnetic field of diverse celestial objects shows good qualitative agreement.

\section{A New Solution Of The Field Equations}

The geometric structure to be used in the present application is an AP-space having spherical symmetry with $n=4$. The structure of this space has been derived by Robertson [8]. The tetrad vector field characterizing this geometric structure is given, in spherical polar coordinates, by:

$$
\left(\lambda_{i}^{\mu}\right)=\left(\begin{array}{cccc}
A & D r & 0 & 0 \\
0 & B \sin \theta \cos \phi & \frac{B}{r} \cos \theta \cos \phi & -\frac{B \sin \phi}{r \sin \theta} \\
0 & B \sin \theta \sin \phi & \frac{B}{r} \cos \theta \sin \phi & \frac{B \cos \phi}{r \sin \theta} \\
0 & B \cos \theta & -\frac{B}{r} \sin \theta & 0
\end{array}\right),
$$

where $A, B$ and $D$ are functions of $r$ only. It has been shown [5] that the geometric structure, given by the matrix (21), has the type FIGI (see section 4). This means that this structure is capable of representing a combined gravitational and electromagnetic weak field outside a spherically symmetric material distribution.

From (10) we get for (21),

$$
\left.\begin{array}{lll}
g_{00}=\frac{B^{2}+D^{2} r^{2}}{A^{2} B^{2}}, & g_{01}=\frac{-D r}{A B^{2}} \\
g_{11}=\frac{1}{B^{2}}, & g_{22}=\frac{r^{2}}{B^{2}}, g_{33}=\frac{r^{2} \sin ^{2} \theta}{B^{2}} .
\end{array}\right\}
$$


Evaluating $E_{\mu \nu}$ in the space (21), and substituting into the field equations (18), we get the following set of differential equations:

$$
\begin{gathered}
\frac{B^{2}+D^{2} r^{2}}{A^{2}}\left[b(r)+\frac{D^{2} r^{2}}{B^{2}} \ell(r)\right]=0 \\
\frac{D r}{A}\left[B(r)+\frac{D^{2} r^{2}}{B^{2}} \ell(r)\right]=0 \\
-\frac{B^{\prime 2}}{B}+\frac{2}{r}\left(\frac{B^{\prime}}{B}+\frac{A^{\prime}}{A}\right)-2 \frac{A^{\prime} B^{\prime}}{A B}-\frac{D^{2} r^{2}}{B^{2}} \ell(r)=0 \\
\frac{D^{2} r^{2}}{B^{2}}\left[-\ell(r)+\frac{A^{\prime \prime}}{A}-\frac{D^{\prime \prime}}{D}+\frac{4}{r}\left(\frac{A^{\prime}}{A}-\frac{D^{\prime}}{D}\right)-2\left(\frac{A^{\prime}}{A}\right)^{2}-\left(\frac{D^{\prime}}{D}\right)^{2}+3\left(\frac{A^{\prime} D^{\prime}}{A D}-\frac{A^{\prime} B^{\prime}}{A B}+\frac{B^{\prime} D^{\prime}}{B D}\right)\right] \\
+\frac{A^{\prime \prime}}{A}+\frac{B^{\prime \prime}}{B}-2\left(\frac{A^{\prime}}{A}\right)^{2}-\left(\frac{B^{\prime}}{B}\right)^{2}+\frac{1}{r}\left(\frac{A^{\prime}}{A}+\frac{B^{\prime}}{B}\right)=0
\end{gathered}
$$

where,

$$
\begin{gathered}
b(r) \stackrel{\text { def }}{=} 3\left(\frac{B^{\prime}}{B}\right)^{2}-\frac{4}{r} \frac{B^{\prime}}{B}-2 \frac{B^{\prime \prime}}{B}, \\
\ell(r) \stackrel{\text { def }}{=} 5\left(\frac{B^{\prime}}{B}\right)^{2}-\frac{8}{r} \frac{B^{\prime}}{B}-2 \frac{B^{\prime \prime}}{B}+\frac{2}{r} \frac{D^{\prime}}{D}-2 \frac{B^{\prime}}{B} \frac{D^{\prime}}{D}+\frac{3}{r^{2}},
\end{gathered}
$$

and the $(')$ represents differentiation with respect to $r$. For these differential equations we have previously obtained three different solutions [5]. A fourth solution, of physical interest, could be obtained as follows. The set of equations (23) -(26) could be written in the more reduced form:

$$
\begin{gathered}
\frac{B^{2}+D^{2} r^{2}}{A^{2}}\left[b(r)+\frac{D^{2} r^{2}}{B^{2}} \ell(r)\right]=0 \\
\frac{D r}{A}\left[b(r)+\frac{D^{2} r^{2}}{B^{2}} \ell(r)\right]=0 \\
f(r)-\frac{D^{2} r^{2}}{B^{2}} \ell(r)=0 \\
s(r)+\frac{D^{2} r^{2}}{B^{2}} K(r)=0
\end{gathered}
$$

where,

$$
\begin{gathered}
f(r) \stackrel{\text { def }}{=}-\left(\frac{B^{\prime}}{B}\right)^{2}+\frac{2}{r}\left(\frac{B^{\prime}}{B}+\frac{A^{\prime}}{A}\right)-2 \frac{A^{\prime}}{A} \frac{B^{\prime}}{B}, \\
s(r) \stackrel{\text { def }}{=} \frac{A^{\prime \prime}}{A}+\frac{B^{\prime \prime}}{B}-2\left(\frac{B^{\prime}}{B}\right)^{2}+\frac{1}{r}\left(\frac{A^{\prime}}{A}+\frac{B^{\prime}}{B}\right), \\
K(r) \stackrel{\text { def }}{=}-\ell(r)+\frac{A^{\prime \prime}}{A}-\frac{D^{\prime \prime}}{D}+\frac{4}{r}\left(\frac{A^{\prime}}{A}+\frac{D^{\prime}}{D}\right)-2\left(\frac{A^{\prime}}{A}\right)^{2}-\left(\frac{D^{\prime}}{D}\right)^{2}+3\left(\frac{A^{\prime}}{A} \frac{D^{\prime}}{D}-\frac{A^{\prime}}{A} \frac{B^{\prime}}{B}+\frac{B^{\prime}}{B} \frac{D^{\prime}}{D}\right)
\end{gathered}
$$

We exclude the solution,

$$
B^{2}+D^{2} r^{2}=0
$$

since it will cause the appearance of imaginary functions. We are interested in solutions that represent combined electromagnetic-gravitational fields. The vanishing of $D$ will 
change the type of space from FIGI to F0GI which represents pure gravity. So, the solution,

$$
D=0
$$

, is to be excluded as well. Now taking $b(r)=0$, the set of differential equations (29)-(32) will reduce to :

$$
\begin{array}{ll}
b(r) & =0 \\
\ell(r) & =0 \\
f(r) & 0 \\
+\frac{D^{2} r^{2}}{B^{2}} K(r) & =0
\end{array},
$$

Using (27), the first equation of the set (36) could be integrated twice to give:

$$
B=\frac{1}{\left(\beta_{1}+\frac{\beta_{2}}{r}\right)^{2}},
$$

where $\beta_{1}, \beta_{2}$ are the constants of integration. Substituting (37) in (28), the second equation of the set (36) can be integrated to give :

$$
D=\frac{\alpha}{r^{\frac{3}{2}}\left(\beta_{1}+\frac{\beta_{2}}{r}\right)^{2}\left(\beta_{1}-\frac{\beta_{2}}{r}\right)} .
$$

where $\alpha$ is a constant of integration. Again using (37) and (33), the third equation of the set (36) can be integrated to give:

$$
A=\beta_{3} \frac{\left(\beta_{1}+\frac{\beta_{2}}{r}\right)}{\left(\beta_{1}-\frac{\beta_{2}}{r}\right)} .
$$

where $\beta_{3}$ is another constant of integration.

Boundary Conditions: The AP-space (21) has spherical symmetry, then it is natural to assume that $\lambda_{i}^{\mu} \rightarrow \delta_{i}^{\mu}$ (in Cartesian coordinates) as $r \rightarrow \infty$. This could be achieved if

$$
\left.\begin{array}{l}
A \rightarrow 1 \\
B \rightarrow 1 \\
D \rightarrow 0
\end{array}\right\} \text { as } r \rightarrow \infty
$$

This implies taking,

$$
\beta_{1}=\beta_{3}=1
$$

For later convenience we take ,

$$
\beta_{2}=\frac{1}{2} m
$$

where $m$ is constant. So, the full solution of the set (36)can be written in the form:

$$
\left.\begin{array}{rl}
A & =\frac{\left(1+\frac{m}{2 r}\right)}{\left(1-\frac{m}{2 r}\right)}, \\
B & =\frac{1}{\left(1+\frac{m}{2 r}\right)^{2}}, \\
D & =\frac{\alpha}{r^{\frac{3}{2}}\left(1+\frac{m}{2 r}\right)^{2}\left(1-\frac{m}{2 r}\right)} .
\end{array}\right\}
$$


It can be shown that solution (43) satisfies the fourth equation of the set (36) without any further conditions.

\section{Physical Interpretation}

In geometric field theories, one is dealing all the time with geometric objects. In order to attribute some physical meanings to these objects, authors usually use the following scheme. They compare the new theory with previous field theories, dealing with the same interaction(s), using certain limiting conditions. For example, the first results supporting the general theory of relativity (GR) have been obtained by comparing the theory with Newton's theory of gravitation, using the conditions of weak static field and slowly moving test particles. As mentioned above, the GFT is an attempt to unify gravity and electromagnetism. It has been compared [11] with both GR and Maxwell's theory of electromagnetism. The results of this comparison have attributed some physical meanings, summarized in Table 2, to certain geometric objects using certain system of units.

Table 2: Physical Meaning of Geometric Objects.

\begin{tabular}{|c|c|}
\hline Geometric Object & Physical Meaning \\
\hline$g_{\mu \nu}$ & Gravitational Potential \\
\hline$C_{\mu}$ & Generalized electromagnetic Potential \\
\hline$F_{\mu \nu}$ & Electromagnetic Field Tensor \\
\hline$T_{\mu \nu}$ & Material-Energy-Stress Tensor \\
\hline
\end{tabular}

where,

$$
\begin{gathered}
F_{\mu \nu} \stackrel{\text { def }}{=} Z_{\mu \nu}-\xi_{\mu \nu}, \\
Z_{\mu \nu} \stackrel{\text { def }}{=} \eta_{\mu \nu}+\zeta_{\mu \nu}, \\
T_{\mu \nu} \stackrel{\text { def }}{=} g_{\mu \nu} \Lambda+\varpi_{\mu \nu}-\sigma_{\mu \nu}, \\
\Lambda \stackrel{\text { def }}{=} \frac{1}{2}(\sigma-\varpi) .
\end{gathered}
$$

In addition to the scheme mentioned above, a new scheme called Type Analysis has been suggested [11], in the context of the GFT, to know the capabilities of an AP-structure to represent physical fields. This procedure is usually applied before solving the field 
equations. It is a covariant procedure since it depends on the values of certain tensors. It can be used to, physically, classify AP-structures to be used for applications (cf. [13] ). Table 3 lists the values of the tensors admitted by a certain geometric AP-structure, the physical meaning of such values and the corresponding codes attributed to these values. It is to be stressed that the first column of this table does not represent conditions on, but values of, the corresponding tensors as calculated from a given AP-structure. The suggested scheme has shown its advantages in several applications (cf. [5], [6], [12]\& [14]).

Table 3: Type Analysis

\begin{tabular}{|c|c|c|}
\hline Tensor Values & Physical Meaning & Code \\
\hline$F_{\mu \nu}=0$ & No electromagnetic field. & $F 0$ \\
\hline$F_{\mu \nu} \neq 0, Z_{\mu \nu}=0$ & Weak electromagnetic field. & $F I$ \\
\hline$F_{\mu \nu} \neq 0, Z_{\mu \nu} \neq 0$ & Strong electromagnetic field. & $F I I$ \\
\hline$R_{. \beta \gamma \delta}^{\alpha}=0$ & No gravitational field. & $G 0$ \\
\hline$R_{. \beta \gamma \delta}^{\alpha} \neq 0, T_{\mu \nu}=0, \Lambda=0$ & Weak gravitational field in free space. & $G I$ \\
\hline$R_{. \beta \gamma \delta}^{\alpha} \neq 0, T_{\mu \nu} \neq 0, \Lambda=0$ & Gravitational field within a material distribution. & $G I I$ \\
\hline$R_{. \beta \gamma \delta}^{\alpha} \neq 0, T_{\mu \nu} \neq 0, \Lambda \neq 0$ & Strong gravitational field within a material distribution. & $G I I I$ \\
\hline
\end{tabular}

The type of the space, under consideration, is written in two parts: The first is one of the codes written in the first three rows of table 3, carrying information about the capability of an AP-structure to represent electromagnetic fields. The second is one of the codes written in the other rows of this table and carrying information about the capability of the same structure to represent gravitational fields. 


\section{$5.1 \quad$ Electromagnetic Quantities}

In the generalized field theory, the vector $C_{\mu}$ has been identified as the generalized electromagnetic potential [3]. The non- vanishing components of this vector, in the space given by (21), have been found to have the values [5]:

$$
\begin{gathered}
C_{0}=\frac{D r}{A}\left(\frac{B^{\prime}}{B}-\frac{D^{\prime}}{D}-\frac{3}{r}\right), \\
C_{1}=\frac{A^{\prime}}{A}+2 \frac{B^{\prime}}{B} .
\end{gathered}
$$

The electromagnetic field tensor $F_{\mu \nu}$ is connected to the electromagnetic potential via the skew-symmetric part of the field equation of the GFT (18),

$$
F_{\mu \nu}=C_{\mu, \nu}-C_{\nu, \mu} \text {. }
$$

From (45) and (46) it is clear that $C_{1}$ will not produce any electromagnetic field, since $A$ and $B$ are functions of $(r)$ only. Then the only non- vanishing components of $F_{\mu \nu}$, in the space (21), are

$$
F_{01}=-F_{10}=C_{0,1}
$$

Using solution (43) to evaluate (44), we get:

$$
C_{0}=\frac{\alpha}{2 r^{\frac{3}{2}}}\left(\frac{1}{\left(1+\frac{m}{2 r}\right)^{2}\left(1-\frac{m}{2 r}\right)}-\frac{4}{\left(1+\frac{m}{2 r}\right)^{3}}\right),
$$

and for (47) we get:

$$
F_{01}=-F_{10}=\frac{\alpha}{4 r^{\frac{5}{2}}\left(1+\frac{m}{2 r}\right)^{4}\left(1-\frac{m}{2 r}\right)^{2}}\left[9-37\left(\frac{m}{2 r}\right)+35\left(\frac{m}{2 r}\right)^{2}-15\left(\frac{m}{2 r}\right)^{3}\right] .
$$

It is clear from(48) and (49) that the constant $\alpha$ has a direct relation to the charge of the system. The vanishing of $\alpha$ will lead to the vanishing of the electromagnetic field. Also, the vanishing of $\alpha$ will change the type of the space from FIGI to F0GI, and this represents pure gravity, as stated above. It is to be considered that (49) represents, in general, the non-vanishing components of a tensor, in a certain coordinate system. Consequently, this tensor will never vanish in any other coordinate system, in the context of the new solution (43). This will be discussed later.

\subsection{The Gravitational Potential}

The symmetric tensor $g_{\mu \nu}$, defined by (10), has been identified in a previous work [3] as the gravitational potential. Using (10) and the solution (43) we get the following non-vanishing components of the gravitational potential:

$$
\left.\begin{array}{l}
g_{00}=\frac{\left(1-\frac{m}{2 r}\right)^{2}}{\left(1+\frac{m}{2 r}\right)^{2}} \quad+\frac{\alpha^{2}}{r\left(1+\frac{m}{2 r}\right)^{2}}, \\
g_{01}=g_{10}=-\frac{\alpha}{r^{\frac{1}{2}}}\left(1+\frac{m}{2 r}\right), \\
g_{11}=\left(1+\frac{m}{2 r}\right)^{4} \quad, \\
g_{33}=g_{22} \sin \theta^{2}=g_{11} r^{2} \sin \theta^{2} .
\end{array}\right\}
$$


To facilitate comparison with general relativity, concerning gravity, we write the indefinite metric of Riemannian space associated with the AP-space(21). This metric is defined in terms of the tetrad vectors via the relation:

$$
\left.\begin{array}{lll}
d s^{2} & = & * g_{\mu \nu} d x^{\mu} d x^{\nu} \\
\text { where } * g_{\mu \nu} & \stackrel{\text { def }}{=} \sum_{i=1}^{4} e_{i}{\underset{i}{\mu}}_{i} \lambda_{\nu} \\
\text { and } e_{i} & = & (1,-1,-1,-1) .
\end{array}\right\}
$$

Using the solution (43), the metric (51) can be written in the form:

$$
d s^{2}=\left[\frac{\left(1-\frac{m}{2 r}\right)^{2}}{\left(1+\frac{m}{2 r}\right)^{2}}-\frac{\alpha^{2}}{r\left(1+\frac{m}{2 r}\right)^{2}}\right] d t^{2}+\frac{2 \alpha}{r^{\frac{1}{2}}}\left(1+\frac{m}{2 r}\right) d r d t-\left(1+\frac{m}{2 r}\right)^{4}\left(d r^{2}+r^{2} d \theta^{2}+r^{2} \sin \theta^{2} d \phi^{2}\right) .
$$

It is of interest to note that the vanishing of $\alpha$ will reduce the metric (52) to the Schwarzschild metric in its isotropic form. This supports the result obtained in the previous subsection (5.1) that $\alpha$ has to be related to the electric charge of the system under consideration.

\section{General Discussion}

(i) It is shown in subsection 5.1 that the vanishing of the constant $\alpha$ will give rise to the vanishing of the electromagnetic field. Also it is shown in subsection 5.2 that the vanishing of $\alpha$ reduces the metric of the associated Riemannian space to the Schwarzschild form. Furthermore, as mentioned above, the vanishing of $\alpha$ will change the type of the space (21) from FIGI to F0GI. These three evidences are consistent with our identification of $\alpha$, that it has to be related to the electric charge of the system. From dimensional consideration, $\alpha$ cannot be directly identified as the charge of the system. Previous experience in Einstein-Maxwell's theory and in classical electrodynamics indicates that: (a) The geometric charge (the charge measured in relativistic units $\mathrm{G}=\mathrm{c}=1$ ) is measured in $\mathrm{cm}$, (b)The electromagnetic field strength and the electromagnetic potential are both linear in the electric charge of the system. In the present treatment it is clear from (50) or (52) that $\alpha$ has the dimension $\mathrm{cm}^{\frac{1}{2}}$; while the electromagnetic field (49) and potential (48) are both linear in $\alpha$ which is consistent with (b). So, to get an agreement between the present results and (a) we should take,

$$
\alpha^{2}=\frac{\epsilon^{2}}{a}
$$

where $\epsilon$ is the geometric charge of the system and $a$ is some constant length to be fixed later.

(ii) Using the coordinate transformation:

$$
\left.\begin{array}{l}
T=t+\psi(r), \\
\psi(r)=\int \frac{\alpha r^{\frac{1}{2}\left(1+\frac{m}{2 r}\right)^{3}}}{r\left(1-\frac{m}{2 r}\right)^{2}-\alpha^{2}} d r, \\
R=r\left(1+\frac{m}{2 r}\right)^{2},
\end{array}\right\}
$$


we can write the metric (52) in the following form:

$$
d s^{2}=\gamma d T^{2}-\frac{d R^{2}}{\gamma}-R^{2} d \theta^{2}-R^{2} \sin \theta^{2} d \phi^{2}
$$

where

$$
\left.\begin{array}{rl}
\gamma & =1-\frac{2 \mu}{R}, \\
\mu & =m+\frac{1}{2} \alpha^{2}, \\
& =m+\frac{1}{2} \frac{\epsilon^{2}}{a} .
\end{array}\right\}
$$

The metric (55), apparently, has the form of Schwarzschild exterior metric in its standard form. This leads to the following interesting physical meaning. The constant $\mu$ can be identified as the total mass of the body causing the curvature of the space. This mass constitutes two parts: The first is the mass $m$ which can be identified as the gravitational mass of the body, while the second is a contribution of the electric charge of the body to its mass. The second part is independent of the sign of the electric charge, as clear from (56).

(iii)Although the metric (55), apparently, has the Schwarzschild standard form, the model represents a combined electromagnetic-gravitational field. This is supported by: (a) The non-vanishing of the electromagnetic field tensor $F_{\mu \nu}$ in the coordinate system $(\mathrm{t}, \mathrm{r}, \theta, \phi)$ as given by (49). The electromagnetic field will never vanish in any other coordinate system, as far as one is using solution (43), since it is represented by a tensor; (b) The appearance of $\alpha^{2}$ term in the metric tensor (52), as $\alpha$ being connected with the electromagnetic field as shown above.

The type of the space used has been found to be FIGI. It is to be considered that the type analysis, by which we know the type of the space, is a procedure independent of the coordinate system used, as it depends completely on tensors of different types. In other words, the type of the space is invariant under general coordinate transformation.

(iv)Another interesting result could be obtained from (56). It is shown that as $\epsilon \neq 0, \mu$ will not vanish. On the other hand, if $\mu=0, m$ will be negative which cannot be accepted. This means that, if the model represents the field of an elementary particle, relations (56) indicate that massless charged particle cannot exist. This result is in agreement with experimental known results. On the other hand, (56) allows for the existence of $m=0$ particles, for which ,

$$
\mu=\frac{1}{2} \frac{\epsilon^{2}}{a}
$$

i.e. particles whose masses are totally electromagnetic in origin [15]. If the model represents an elementary charge ( an electron or a positron), and assuming that $M$ is the mass of the electron in gm and $Q$ is its charge in e.s.u. then

$$
\mu=\frac{G M}{c^{2}} \quad, \quad \epsilon=\frac{c^{\frac{1}{2}} Q}{c^{2}},
$$

where $c$ is the speed of light, and $G$ is the gravitational constant. Substituting from (58) into (57), we get:

$$
2 a=\frac{Q^{2}}{c^{2} M}
$$


Substituting the numerical values [16] of $Q, M$ and $c$, we get from (59),

$$
2 a=2.81794 \times 10^{-13} \mathrm{~cm}
$$

this length coincides with the classical radius of the electron. So, in general $2 a$ can be identified as the radius of a particle whose mass is electromagnetic in origin.

(v) It may be of interest to interpret (56) using classical electrodynamics. A charged particle of radius $2 a$ and charge $Q$ will have a self potential energy (cf. [17] ):

$$
\left.\begin{array}{l}
\xi=\frac{Q^{2}}{4 a} \quad(\text { in } \mathrm{cm} \text { gm sec units }), \\
\xi=\frac{\epsilon^{2}}{4 a} \quad(\text { in relativistic units }) .
\end{array}\right\}
$$

Now, (56) can be interpreted in view of (60) as follows: apart from the factor 2, the gravitational mass of any charged particle will be increased by an amount equal to the self potential energy of the field produced by the charge. An interpretation similar to the above one has been obtained by Florides [18], by applying Moller's theory on energy [19] to the well known Riessner-Nordstrom solution ; and then studying the motion of a neutral particle in the field of a central charged body. From the metric point of view, one of the differences between the present work and that of Florides [19] is that we have obtained a solution whose associated Riemannian space has the metric of the Schwarschild exterior form, using which we get the result.

\section{Conclusion}

The constants of integration have been fixed using certain boundary conditions. The comparison with classical known results has given rise to the identification of the constant $m$ as the gravitational mass of the body, the constant $\epsilon$ as its geometrical charge, and the constant $a$ as half its radius. The appearance of the two constants $m$, and $\alpha\left(=\frac{\epsilon}{a^{\frac{1}{2}}}\right)$ in both electromagnetic and gravitational quantities shows the interaction between the two fields.

The solution allows for the existence of a charged particle whose total mass is electromagnetic in origin, and independent of the charge sign. On the other hand, the same solution prevents the existence of massless charged particles. In general, the solution shows that the mass of any body, with net electric charge, is made of two contributions: One is its gravitational mass and the other is due to its net electric charge.

Finally, it is of interest to note that the metric of the Riemannian space is not sufficient to give a complete indication of the physical content of the field. It is more convenient to use the procedure known as the type analysis in order to get more insight on the physical fields that a certain space is capable of representing. 


\section{REFERENCES}

[1] R.Adler, M.Bazin, and M.Schiffer,Introduction to General Relativity (McGraw-Hill 1975) 2nd edition, p.486.

[2] F.I.Cooperstock, and V.De La Cruz, Gen.Relativ.Gravit.9 , 835 (1978).

[3] F.I.Mikhail, and M.I.Wanas, Proc.R.Soc.London A 356,471 (1977).

[4] M.I.Wanas, Nuovo Cimento 66B,145 (1981).

[5] M.I.Wanas, Int.J.Theor.Phys. 24, 639 (1985).

[6] F.I.Mikhail, M.I.Wanas,and A.M.Eid, Astrophys. Space Sci.228 , 221 (1995).

[7] A.Einstein, Math. Annal. 102, 685 (1930).

[8] H.P.Robertson, Ann.Math.Princeton(2),33,496 (1932).

[9] F.I.Mikhail, Ph.D. Thesis, London University (1952).

[10] N.L.Youssef and A.M.Sidahmed (2006) gr-qc/0604111.

[11] F.I.Mikhail , and M.I.Wanas, Int.J.Theor.Phys.20, 671 (1981).

[12] M.I.Wanas, Astrophys. Space Sci.154 , 165 (1989).

[13] M.I.Wanas (2001) Proceedings of the 11th National Conference on

Finsler, Lagrange and Hamilton Geometries, (Bacau, 2000),

Cercet.Stiin.Ser.Mat 10:,297-309,2001, eds. V.Blanuta and Gh.Neagu; gr-qc/0209050

[14] M.I.Wanas Astrophys. Space Sci.127, 21 (1986)

[15] M.I.Wanas (1987) ICTP preprint IC/87/397.

[16] C.W.Allen, Astrophysical Quantities (London 1973) 3rd edition,p.14, 15.

[17] L.D.Landau ,and E.M.Lifshitz, The Classical Theory of Fields (Pergamon 1975) 4th edition, P.89.

[18] P.S.Florides, Proc.Camb.Phil.Soc.58, 110 (1962).

[19] P.S.Florides, Proc.Camb.Phil.Soc.58, 102 (1962). 